\title{
LA BELLA DE IJUAN RUIZ TENÍA LOS OJOS DE HURÍ
}

Gustavo Adolfo Bécquer, con su galantería legendaria, consuela a una muchacha que dio en pensar que sus ojos verdes desmerecían su rostro agraciado:

\author{
Porque son niña, tus ojos \\ verdes como el mar, te quejas; \\ verdes los tienen las náyades, \\ verdes los tuvo Minerva, \\ y verdes son las pupilas \\ de las hurís del profeta.
}

Se equivocaba Bécquer. Las huríes del paraíso musulmán nunca tuvieron los ojos verdes, que hubieran horrorizado de seguro a los seguidores del Profeta, tan poco proclives a los ojos claros ${ }^{1}$. Pero no inculpemos de ignorancia estética al cortesísimo poeta andaluz, que no hacía otra cosa que aleccionar a su niña en el apre-

${ }^{1}$ Los ojos azules o verdes se suelen asociar en la literatura árabe a lo demoniaco o grotesco. Todavía leemos en los códices aljamiado-moriscos los últimos ecos de esta tradicional antipatía estética: en la leyenda de Alejandro Magno el temible color azul se le atribuye a los ojos de la gente de Amoazón, mientras que, en otra leyenda, se asegura que el día del Juicio, el justiciero Alidachel tornará al pecador, aunque sea blanco, en un negro de ojos zarcos. (Cf. F. Gullén Robles, Leyenda de Alejandro Magno, Imprenta del Hospicio Provincial, Zaragoza, 1888, p. 252; y Leyendas moriscas, Imprenta y Fundición de M. Tello, Madrid, 1886, t. 3, p. 338). Claro que ha habido importantes excepciones a la regla en las letras árabes: basta recordar con cuánto entusiasmo la poesía culta de Al-Andalus celebró los cabellos de oro y los ojos claros, característicos, de otra parte, de la familia omeya. Sin embargo, debemos admitir que lo hizo por excepción. El rechazo generalizado de los ojos claros se perpetúa hasta el día de hoy en muchos países musulmanes: aún algunos mercaderes de zoco actúan con recelo si el primer cliente de la mañana tiene los ojos azules o verdes. 
cio de sus ojos marinos: es que el color de los ojos de las huríes ha resultado un verdadero enigma para Occidente, que no ha sabido qué hacer - ni cómo traducir adecuadamente- el misterioso resplandor de las pupilas de las compañeras sempiternas de los musulmanes bienaventurados.

Abrimos el Libro Revelado y encontramos que las azoras XLIV, 54 y LII, 20 desmienten en seguida a nuestro entusiasta Bécquer al describirnos a las compañeras paradisíacas de los elegidos como " "بح siguiente versión inglesa al pasaje coránico: se trata de compañeras que tienen "beautiful, big, lustrous eyes"2. Los ojos de las huríes resultan misteriosamente incoloros, una vez más, en la versión española del Corán de Juan Vernet: "Los casaremos con mujeres de ojos rasgados, huries"'3. Vernet, en lugar de aludir al resplandor de los ojos, incorpora el término con el cual se conocerán tanto en Oriente como en Occidente las hermosas vírgenes del Paraíso islámico poseedoras de esa mirada luminosa e incitante: huríes. Pero es que el término mismo de "hurí" daría la pista a un lector de árabe acerca del color real de las pupilas de las agraciadas féminas del trasmundo: se trata de ojos "respiandecientes" o "relucientes" justamente por el fuerte contraste que se establecc entre el negro azabache de la pupila y el blanco purísimo de la córnea. Eso es precisamente lo que significa la palabra hūr $(,-5)$, de donde proviene el apelativo de hīuinga ( de hūr (, ) -hurí- aplicado como sobrenombre constante de las vírgenes del Paraíso ${ }^{4}$. A. Yusuf Ah elabora el sentido estético y espiritual del término:

Hür implies the following ideas: (1) purity; possibly the word Haw$w \bar{a} r i \bar{u} n$, as applied to the first disciples of Jesus, is connected with this root; (2) beauty, especially of eyes, where the intense white of the eye balls stands out against the intense black of the pupil, thus giving the appearance of lustre, and intense feeling, as opposed to dullness or want of expression; and (3) truth and good-will ${ }^{5}$.

2 The Holy Qur'an, Text, Transiation and Commentary by A. Yusuf Ali, McGregor and Werner, 1946, pp. 1352 y 1435.

${ }^{3}$ El Corán, trad., introd. y notas de J. Vernet, Planeta, Barcelona, 1963, p. 558 .

${ }^{4}$ Cf. Arabic-English Dictionary, ed. by J. M. Cowan, Spoken Languages Services, Ithaca, NY, 1976, p. 212.

${ }^{5}$ Ibid., p. 1352. 
Si dejamos a un lado las cualidades morales asociadas al término $h \bar{u} r^{6}$, encontramos en el comentario del estudioso las claves principales de la apariencia de este modelo estético de ojos: el contraste del blanco y el negro los hace parecer lustrosos o resplandecientes al que los mira. De ahí que muchas veces estos ojos de la hembra árabe paradigmática — tan ajenos a la sensibilidad occidental- se traduzcan a las lenguas occidentales de manera aproximativa e imprecisa. Algunas veces el traductor nos indicará que se trata de ojos en los que contrastan fuertemente el blanco y el negro; otras, hará referencia simplemente al color negro de los ojos, y otras, los describirá como ojos "relucientes", omitiendo por lo general cualquier información acerca de su color específico. De ahí a imaginar que estos ojos incoloros debían ser verdes, como hizo Bécquer, no había más que un paso: sólo a un buen conocedor de la lengua y de la cultura estética árabes le sería dado el saber que el apelativo de "resplandecientes" apuntaba hacia una mirada lustrosa por la intensa antítesis cromática con la que estos ojos, enormes y rasgados, estaban agraciados.

Pero los ojos de las huríes y de las féminas arquetípicas de los poetas árabes fueron indiscutiblemente negros y no verdes ${ }^{7}$. En un arrebato apasionado, un poeta anónimo cuyos versos usurpa el Šeyj Nefzāīi admite que: "Somos un pueblo tan fuerte que podemos doblar el acero,/pero sin embargo sucumbimos a los grandes ojos negros"'8. (Por cierto que todavía se escucha en España un fandango que repite la rendida celebración en términos parecidos: "ni los corregidores/tienen poderes/para los ojos negros/de las mujeres". .) Negros también serían los ojos que celebra Ibn 'Arabī, esta vez un poeta místico, que, buen espiritual musulmán al fin, no se inhibe de celebrar la belleza humana en su Tarŷumàn al-ašwāq o Intérprete de los deseos. Las doncellas que evoca tienen rostros brillantes y asesinan de amor precisamente con sus ojos

${ }^{6}$ La raíz árabe 'hūur, tiene, naturalmente, más sentidos. Cowan apunta a otros como "disminuir; cambiar; modificar; blanquear; transformar; discutir", entre otros (id.).

${ }^{7}$ Sobre la apariencia física de los personajes de la literatura árabe y aljamiado-morisca, cf. mi ensayo "La estética del cuerpo entre los moriscos del siglo XVI o de cómo la minoría perseguida pierde su rostro", en Augustin Redondo (ed.), Le corps dans la societé espagnole des xvi et xvit siècles, Publications de la Sorbonne, 1990, pp. 335-348.

${ }^{8}$ The Glory of the Perfumed Garden. The Missing Flowers, Neville Spearman, London, 1975, p. 200. El traductor, que vierte el texto al inglés en una versión muy profesional, sólo se firma "H. E. J.", incómodo de seguro por el contenido erótico de la obra de Nefzāwī. 
negros $^{9}$. R. A. Nicholson traduce acertadamente el pasaje árabe

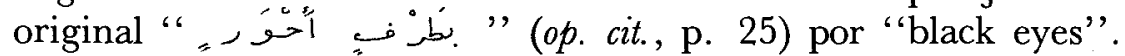
Si fuéramos a traducir los versos con mayor exactitud, tendríamos que aludir a unos "ojos en los que contrasta el negro de la pupila con el blanco de la córnea", ya que, como dejamos dicho, eso es lo que implica la raíz hūr que emplea Ibn 'Arabī en su texto árabe original.

Y ese es precisamente el contraste cromático que respeta Sir Richard Burton en su traducción inglesa del Jardín perfumado del Seyj Nefzāwī. Cuando el erotólogo establece su ideal estético femenino, celebra la calidad de $h \bar{u} r$ que deben tener, invariablemente, los ojos de su bella. Burton, sabiendo que el término es difícil de traducir en las lenguas europeas, aclara su sentido literal: "she will have eyebrows of Ethiopian blackness, large black eyes, with the white of them very limpio" "10. Con este mismo contraste deslumbrante del blanco y el negro juega el anónimo autor del Speculum al foderi, un sorprendente manual de higiene sexual que se transforma de súbito, en sus capítulos finales, en un tratado erótico en toda forma. (Por cierto que nuestro misterioso médico, que escribió hacia el siglo xIv, se cuida de no dar noticia en el índice de su códice del contenido francamente sexual de estos capítulos últimos.) Cada vez sabemos más acerca de estos textos eróticos, refundiciones obvias de originales árabes, que circularon en España más ampliamente de lo que hemos querido admitir hasta ahora. Franciseo Márquez Villanueva no fue sino un profeta de la erotología hispánica orientalizada cuando teorizó en su espléndido ensayo "Las lecturas del Deán de Cádiz""11 que lo que solía leer el capitular gaditano de la cantiga de Alfonso X no eran smo códices erotológicos que debían más a la cultura oriental que a Ovidio ${ }^{12}$. Pero no perdamos de vista lo que nos interesa aquí:

${ }^{9}$ Tarjuman al-Ashwaq. A Collection of Mystical Odes, tr. by R. A. Nicholson, Royal Asiatic Society, London, 1911, p. 92.

10 The Perfumed Garden, Castle Books, New York, 1964, p. 21.

11 "Las lecturas del Deán de Cádiz en una cantiga de mal dizer", en Studies in the "Cantigas de Santa Maria"': Art, Music and Poetry, Proceedings of the International Symposium on the Cantigas de Santa María, ed. by I. J. Kotz and J. E. Keller, Hispanic Seminary of Medieval Studies, Madison, WI, 1987, pp. 329-354.

12 Por mi parte, acabo de editar el texto completo del manual erótico que un anónimo morisco expulsado a Túnez en 1609 incluye en un extenso códice misceláneo que hoy conocemos como el ms. S-2 de la Biblioteca de la Real Academia de la Historia de Madrid. Se trata de un texto verdaderamente inusitado e importantísimo para la historia de las ideas en España: por primera 
los ojos de hurí de las féminas árabes —ahora, arabizadas- que nuestro anónimo autor catalán adapta a su propia cultura estética "europea". Instruye al lector en lo tocante a los distintos atributos que por fuerza ha de exhibir su "bella", que nos resulta, a todas luces, más oriental que catalana:

En cuanto a la nobleza y la belleza de las mujeres, se trata de que tengan cuatro cosas muy negras: el pelo, las cejas, las pestañas y los ojos; cuatro cosas muy coloradas: las mejillas, la lengua, las encías y los labios; cuatro muy blancas: el rostro, los dientes, el blanco de los ojos y las piernas; cuatro muy estrechas: los orificios de la nariz y de los oídos, la boca, los pechos y los pies; cuatro muy delgadas: las cejas, la nariz, los pechos y las nalgas; cuatro muy redondas: la cabeza, el cuello, los brazos y las piernas; y cuatro muy perfumadas: la boca, la nariz, las axilas y el coño ${ }^{13}$.

No es arriesgado pensar que el autor de este curioso " $K \bar{a} m a$ Sưtra catalán" (el término es nuestro) está adaptando algún original árabe ${ }^{14}$ en el que ha aprendido a gustar, entre muchas otras cosas, del contraste del blanco y el negro en los ojos de una mujer hermosa. Exactamente la misma característica estética exige la Doncella Teodor, aquella marisabidilla en amores de las Mil y una noches refundida en castellano, a quien quiso inútilmente avergonzar su interlocutor al interrogarla en lo tocante a erudición erótica. La virginal Tudur lo deja sorprendido, pues, buena árabe a fin de cuentas, pertenecía de lleno a una larga tradición teórica

vez se celebra el placer sexual al margen de la pornografía y de la culpabilidad religiosa. Para colmo, el anónimo autor del S-2 cita a sus autoridades musulmanas pertinentes, pero las entrevera de sonetos de Lope de Vega. El mestizaje cultural de este manual, que he llamado el "Kama Sütra español" por falta de un mejor título, es flagrante y hará correr mucha tinta en el futuro porque viene a darnos una idea completamente distinta de lo que fue la literatura española del Siglo de Oro. No sólo no la hemos estudiado adecuadamente, sino que ni siquiera la hemos editado en su totalidad.

${ }^{13}$ Citamos por la versión española de Teresa Vicéns, Speculum al joder. Tratado de recetas y consejos sobre el coito, Pequeña Biblioteca Calamvs Acriptorius, 1978, p. 47. En 1917, Ramon Miquel y Planas publica el Speculum en una edición de tirada muy restringida y dirigida a bibliófilos, que parece no haber sobrevivido. Michael Solomon acaba de editar la versión bilingüe catalano-inglesa para el Hispanic Seminary of Medieval Studies de Madison, WI (1990).

${ }^{14}$ Aún no sabemos a ciencia cierta a quién traduce, aunque sí hay que tomar en cuenta que comienza su códice citando respetuosamente a una autoridad árabe de nombre "Albafumet". 
en materia de amores, que sabía manejar a la perfección:

Digo que [la mujer debe ser] luenga en tres, que sea luenga d'es$\mathrm{ta}[\mathrm{n}] \mathrm{do}$, e que aya el cuello largo e los dedos luengos; [e] blanca en tres: el cuello blanco e los dientes blancos e lo blanco de los ojos blanco; e pryeta en tres: cavellos pryetos e las cejas pryetas e lo de los ojos negro, que sea pryeto; e bermeja en tres: labros, maxillas, enzías; e pequeña en tres: boca pequeña, narizes pequeñas e los pies pequeños; e ancha en tres: ancha de caderas e ancha de espaldas e ancha la fruente; e que sea muy plazentera a su marydo e muy ayudadera, e que sea pequeña de hedat ${ }^{15}$.

Salta pues a la vista que los ojos en contraste de blancura y oscuridad son el sine qua non de la bella por excelencia de la tradición estética árabe, que no dejó de reflejarse en las refundiciones y en los textos arabizantes del medioevo español. Lo que estos adaptadores peninsulares tendrían en mente era el término hūr, que se prestaba fácilmente a esta subdivisión en dos colores que debían tener inexorablemente los ojos, seguramente grandes y rasgados, de la fémina en cuestión.

Pero es evidente que el término hür no se presta a una traducción o refundición fácil. Se trata de unos ojos difíciles de concebir -incluso, de gustar- en Occidente. Los poetas de Al-Andalus, como era de esperar, celebraron a su vez esos ojos de tonos contrastantes, y Henri Pérès, en su versión francesa de estos poemas (que Mercedes García Arenal a su vez vierte al español), opta por una solución alterna: llamar a los ojos de tono bicolor no ya negros (ni blancos y negros) sino, directamente, "ojos de huríes". Así procede Pérès, por ejemplo, cuando traduce los versos de Baššăr ibn Burd o de Ibn Hazm ${ }^{16}$. No es la primera vez, por cierto, que un traductor del árabe da ese rodeo para salir de apuros: el anónimo autor del ms. S-2 de la Biblioteca de la Real Academia de la Historia de Madrid, cuando describe a las vírgenes del $\mathrm{Pa}$ raíso coránico, evita llevar a cabo una descripción minuciosa de sus ojos incitantes al llamarlas, sencillamente, "haurías" ${ }^{17}$. Nues-

${ }^{15}$ Citamos por la Crest. de R. Menéndez Pidal, apud Libro de buen amor del Arcipreste de Hita, ed. de Jacques Joset, Espasa-Calpe, Madrid, 1981, p. 164. Advertimos por cierto que la versión de la Historia de la Doncella Teodor de Walter Mettman (Franz Steiner Verlag GMBH, Wiesbaden, 1962, p. 119), ofrece variantes al pasaje que acabamos de citar.

${ }^{16} \mathrm{Cf}$. Henri PÉRÈs, El esplendor de Al-Andalus, trad. Mercedes García Arenal, Hiperión, Madrid, 1983, pp. 406 y 408.

${ }^{17}$ Así, por ejemplo, en el f. $246 \mathrm{v}^{\circ}$. Este morisco es el autor del "Kāma Sütra 
tro refugiado en Túnez sería perfectamente consciente que con este apelativo sus lectores bilingües (aquellos lastimados moriscos relativamente arabizados que llegaron a las playas de Berbería a principios del siglo xvil) habrían de entender que las compañeras de los bienaventurados recibían este apelativo precisamente por su sugestiva mirada en fuerte contraste de negro y blanco.

La mirada de estas huríes del Paraíso y de su contrapartida humana, las féminas particularmente favorecidas por la naturaleza, debía ser, como dejamos dicho al principio, una mirada resplandeciente y particularmente luminosa. Hay muchos traductores del árabe que optan por destacar tan sólo esta última característica de los ojos de hurí de las féminas árabes: su brillo, al margen del color - mejor, de los colores contrastantes- que producen este lustre especial en la mirada. Ya indicamos que es precisamente en estos términos que muchos adaptadores del Corán a las lenguas occidentales intentan comunicar a sus lectores cómo eran los ajos de las compañeras de los ele gidos. Aludían tan sólo a su resplandor luminoso y dejaban sin traducir su verdadero color: así vimos qué hizo A. Yusuf Ali en su cuidadosa versión inglesa del Libro Revelado. También los refundidores de otros textos en los que se incorporan los ojos ideales de los árabes - tanto los humanos como los divinales - optan por privilegiar en su descripción este destello resplandeciente de las miradas de las mujeres de hermosura paradigmática, aun cuando a veces aludan, por razones de exactitud, a su color contrastante. Así, L. Bercher y G. H. Bousquet dan la siguiente versión de la esposa idealizada que describe Algazel a los candidatos al matrimonio: " $h$ 'awar veut dire: blancheur. La femme h'awrâ est celle dont le blanc et le noir de l'œil sont particulièrment éclatants. . .',18 Otros traductores evitan el circunloquio y, como Yusuf Ali, evitan aludir a los colores en dramática oposición que dotan de lustre a las miradas de sus bellas, y sólo dan noticia escueta del brillo resultante. Un personaje de la Gloria del jardín perfumado de Nefzāwī pormenoriza a su dama, quien, entre otros atributos, debe exhibir, en la versión del refundidor inglés, "eyebrows [...] as perfect as if they had been traced with a pen or blackened with charcoal and they arch over eyes like those of a graceful fawn. They dazzle the onlooker

español" al que acabo de aludir en la nota 10, aunque el pasaje que ahora cito no pertenece a su tratado erotológico sino a una descripción del Paraíso.

${ }^{18}$ GHazÂL̂̀, Le livre de bons usages en matière de mariage. (Extrait de l'Ihya' oulum ed-Din ou Vivification de las sciences de la foi, A. Maisonneuve, Paris-J. Thornton and Son, London, 1953, p. 62. 
and defy his powers of description..."19. Nos hemos quedado pues en el relámpago fulgúreo de estas pupilas inigualables. Ya no se nos indica su color, sino sólo cómo relucen ante el afortunado admirador que las contempla. Privilegiar la cualidad más dramática del brillo que debe tener la mirada hermosa por excelencia a expensas del color ha sido una buena solución intermedia de este sagaz traductor inglés.

Fue, precisamente, la misma solución que ya había utilizado el Arcipreste de Hita en el siglo xiv. A todos nos consta hoy que la bella de Juan Ruiz era "toda problemas" porque, con sus "dientes apartadiellos" y sus caderas "anchietas" constituía un paradigma estético prácticamente irreconocible en Occidente. Y clásico en Oriente. Añadamos una curiosidad más a las que ya apuntaron, en un célebre ensayo, Dámaso Alonso y Emilio García Gó$\mathrm{mez}^{20}$. El Arcipreste, que tanto pormenoriza los atributos de su dama, no nos indica el color que deben tener sus pupilas. Dando un misterioso (¿irónico?) mentís a aquellos ojos azules o verdes que ya se cantaban con obsesión en el resto de la poesía europea, nos alecciona en el reguste estético de unos "Ojos grandes, someros, pintados, reluzientes,/e de luengas pestañas, bien claras, pareçientes"'21. Resulta algo curioso el hecho que Don Amor, que es aquí quien dicta el ideal estético femenino, pase por alto el cromatismo de estos ojos paradigmáticos que le propone al Arcipreste: un europeo lo echaría de menos. Pero no es mucho sospechar que los lectores - mejor, los oidores- del célebre librete del Arcipreste sabrían muy bien cómo gustar de estos ojos "reluzientes": su lustre se debería sin duda al fuerte contraste del negro del iris con el blanco de la córnea. No había nada más que indicar al buen conocedor, ni dar más rodeos en la descripción física de aquellos ojos de la bella: la aclimatación española del término hür les sería clara a aquella mozarabía que escuchaba con tanto agrado y con tanto savoir faire al Arcipreste. Y que de seguro lo supo entender mejor que nosotros.

Advirtamos que Don Amor subraya el brillo resplandeciente de estos misteriosos ojos de su hembra hermosa: deben ser no sólo "reluzientes" sino "pintados". Curiosos adjetivos. Este últi-

${ }^{19}$ Op. cit., pp. 208-209. Ya dejamos dicho que el traductor nos oculta su nombre bajo las iniciales de H. E. J.

20 "'La bella de Juan Ruiz, toda problemas", en De los siglos oscuros al Siglo de Oro, Gredos, Madrid, 1964, pp. 86-99.

${ }^{21}$ Citamos el verso 433 por la edición citada de J. Joset, p. 165. 
mo término lo entiende J. Joset ${ }^{22}$ como "brillantes", con lo que una vez más, estamos ante la celebración del extraño resplandor de la mirada agarena de la guapa de Juan Ruiz. Es curioso apuntar que me he encontrado con estos "ojos pintados" justamente en contextos hterarios altamente "sospechosos" por su innegable arabización: en códices aljamiados. En el tratado astrológico incluido en el ms. Junta XXVI, el anónimo autor describe los ojos de los varones nacidos bajo el signo de Leo como "ojos pintados" 23 . El guapo leonino debió tener ojos brillantes por ser muy negros y estar en contraste con el blanco de la esclera ${ }^{24}$. Es fuerza admitir que el anónimo morisco y Juan Ruiz compartían un mismo vocabulario cuando se disponían a describir los ojos orientales de sus personajes: debían estar traduciendo el mismo concepto árabe de $h \bar{u} r^{25}$.

Esos ojos de la problemática bella de Juan Ruiz deberían ser, naturalmente, enormes, y estar enmarcados por unas pestañas profusas y una cejas muy bien delineadas: "luengas, altas en peña", (432c). Claro que parecen unos ojos agarenos típicos. Pero hay que añadir otro dato a la pintura de estos ojos que le resultaron al Arcipreste paradigma de toda hermosura: Don Amor los llama "someros" (433a). Según J. Joset, estos ojos son "salientes", por oposición a los ojos fondos o hundidos de la serrana fea que vitupera estéticamente nuestro poeta ${ }^{26}$. También los árabes celebraron esta curiosa condición, con una pequeña variante que quién sabe si estaba también en la mente de Juan Ruiz. Nefzāwī exige que los ojos de su dama sean "someros" porque "[they enhance] with their curves all that lies bellow them" 27 . Es decir, que "presiden" - -por así decirlo - todo lo que queda bajo ellos en el rostro de la hermosa.

Todo parece indicar que Dámaso Alonso no andaba descaminado cuando reflexionaba que en la dama del Arcipreste había

${ }^{22} \mathrm{Id}$

${ }^{23}$ Cf. mi estudio al respecto, escrito en colaboración con LuISA PIEMonTESE y Claire MarTin, "Un morisco astrólogo, experto en mujeres", $N R F H$, 36 (1988), 261-276.

${ }^{24}$ Otros manuscritos aljamiados traen la variante de "ojos alcoholados", que podrían haber sido ojos pintados con carboncillo o al-kuhul, u ojos que, por su contraste de colores blanco y negro, parecerían pintados con carboncillo.

${ }^{25}$ Debo señalar que mi respetado colega J. JoseT tiene una visión distinta acerca de esta posible impronta árabe por parte de Juan Ruiz. Cf. su estudio Nuevas investigaciones sobre el "Libro de buen amor", Cátedra, Madrid, 1988.

${ }^{26}$ Ed. cit., p. 165.

27 The Glory..., p. 209; las cursivas son mías. 
un grupo de elementos que se podrían considerar ajenos o al menos extraños a la cultura occidental, pero que en cambio, "todos [...] han surgido con ímpetu, irrestrañables, en el primer par de sangraduras que (gracias a García Gómez) hemos hecho en la vena árabe"'28. Esa vena, que yo misma he sangrado anteriormente en otros ensayos en torno al Arcipreste ${ }^{29}$, ha dado mucho de sí y habrá de dar aún más cuando dejemos de desdeñar la contextualidad literaria árabe en nuestras exploraciones acerca del Libro de buen amor. No pueden parecernos extrañas en un poeta que sabe rimar en un árabe dialectal impecable ${ }^{30}$. Por el momento cabe adelantar que aquellas "enzías bermejas" de las que no hubiera hecho gala jamás Melibea y mucho menos Madonna Laura, son sin embargo requisito sine qua non de las bellas del Islam, desde la de Nefzāwī hasta las que vimos avalaban el anónimo autor capitán del Speculum al foderi y la erudita Tudur. Tan deseables eran las encías bermejas que las hijas de Agar masticaban unas nueces especiales que les teñían las encías de un color todavía más rojo. Una vez más, el gusto por los colores en dramático contraste, ahora, entre el rojo encendido y el blanco de los dientes, que debían ser para estos erotólogos del Islam invariablemente "agudillos'”31, tal como los quería a su vez nuestro Arcipreste. Y la nariz, también al más estricto gusto semita: "afilada" la exige Juan Ruiz, y Nefzāwì, en términos más poéticos, la imagina “'tan afilada como la punta de una espada bruñida"'32. Claro que los expertos del Islam desnudan a su bella y celebran sus encantos

28 Art. cit., p. 462.

29 "Juan Ruiz y el Śeyj Nefzāwī «elogian» a la dueña chica", $L T$, 1 (1987), 461-472; "Sobre el signo astrológico del Arcipreste de Hita', en Huellas del islam en la literatura española. De Juan Ruiz a Juan Goytisolo, Hiperión, Madrid, 1985 y 1989, pp. 43-58. Para una bibliografía de los elementos arabizantes del Libro de buen amor y, en particular, de la bella de Juan Ruiz, véanse estos ensayos y la citada edición de Jacques Joset (sobre todo las pp. 163-166).

${ }^{30}$ Así, en los versos en los que describe cómo la mora desprecia el mensaje del Arcipreste que le trae Trotaconventos (vv. 1508 ss.). Es curioso apuntar en este sentido que la mora, personaje que solía ser considerado precisamente como un sex symbol en la época, es la única hembra que pone orden al lascivo Arcipreste. ¿Estamos ante otra broma de nuestro inquieto poeta, que estaría rindiendo un curioso e inesperado homenaje a estas mujeres árabes que sin duda alguna le fueron familiares en su vida personal en aquella España que aún no había asfixiado su orientalidad cultural?

31 Así, por ejemplo, en Nefzāwī "teeth that are sharply cut and as white as pearls"' (The Glory..., p. 209).

$32 \mathrm{Id}$. 
ocultos, para los que también hay reglas precisas: aquí el Arcipreste siente que le pesa su herencia cultural cristiana y, más púdico, se contenta con advertir al lector "puna de aber muger que la vea sin camisa" (435c). Con todo, salta a la vista que el enigmático poeta peninsular sigue las lecciones magisteriales de los erotólogos árabes, que, como dejamos dicho, debieron haber circulado en España mucho más de lo que hemos querido admitir hasta ahora. Parece irónico el hecho de que Don Amor invoque a Ovidio y al Liber Pamphilus antes de comenzar su alocución estética en torno a esta bella "inclasificable": nada de lo que enseña a su buen alumno el Arcipreste se encuentra allí, sino precisamente en los libros de erotología oriental. Pero ya sabemos cuánto ha citado en falso el travieso Juan Ruiz: sus alusiones espúreas parecen ser la regla y no la excepción ${ }^{33}$.

Los "problemas" que aquejan a la bella de Juan Ruiz se van esfumando desde contextos literarios árabes: todo parece indicar que sus ojos extrañamente incoloros son los ojos lustrosos de las bellas del Islam, en fuerte contraste esos magníficos ojos heredados de las hijas de Agar, y hasta el mexicano Agustín Lara no se ha inhibido de cantar a la "mujer que conserva el embrujo de los ojos moros". Y no sabría el autor de la célebre pieza "Granada" que estos ojos moros de verdad envolvieron en su legendario embrujo (sihr) a los hispanoárabes a lo largo de toda la Edad Media. Si el embrujo antiguo de aquellos ojos ha durado hasta hoy, no parece mucho admitir que, allá por el siglo xIV, todavía la bella de Juan Ruiz, más agarena que española, mirara sugestivamente al Arcipreste con sus ojos resplandecientes de hụ $\bar{r} \overline{\mathrm{d}} \mathrm{del} \mathrm{Pa}$ raíso.

Luce López-Baralt Universidad de Puerto Rico

${ }^{33}$ Para las citas equivocadas de Ptolomeo, que una vez más hay que referir a fuentes árabes, cf. mi citado ensayo "Sobre el signo astrológico del Arcipreste de Hita". 
